\title{
Cerebral Oxygenation during Pediatric Cardiac Surgery: Identification of Vulnerable Periods using Near-infrared Spectroscopy
}

\author{
${ }^{1}$ Mehul Mange, ${ }^{2}$ Manjula Sarkar
}

\begin{abstract}
Objective: Neurologic sequelae remain a well-recognized complication of pediatric cardiac surgery. The aetiology of neurologic injury is almost certainly multifactorial, imbalance between cerebral oxygen supply and demand is likely to play an important role. We sought to measure regional cerebral oxygenation in children undergoing cardiac surgery using nearinfrared spectroscopy to ascertain such vulnerable periods.
\end{abstract}

Materials and methods: This study is an observational study of 18 children (median age 1.3 years) undergoing cardiac surgery. Regional cerebral oxygenation was monitored using the INVOS3100 cerebral oximeter and related to hemodynamic parameters at each stage of the procedure.

Results: Prior to the onset of bypass, 10 patients had a decrease in regional cerebral oxygenation, reaching a saturation less than $35 \%$ in 5 cases. The most common cause was handling and dissection around the heart prior to and during caval cannulation. With institution of bypass, regional cerebral oxygenation increased. Discontinuation of bypass caused a precipitous decrease in regional cerebral oxygenation in three patients, reaching less than $40 \%$.

Conclusions: These observations suggest that the pre- and early post-bypass periods are vulnerable times for provision of adequate cerebral oxygenation. Near-infrared spectroscopy is a promising tool for monitoring $\mathrm{O}_{2}$ supply/demand relationships especially during circulatory arrest.

Keywords: Cardiopulmonary bypass, Cerebral oximetry, Children, Deep hypothermic circulatory arrest, Near-infrared spectroscopy.

How to cite this article: Mange M, Sarkar M. Cerebral Oxygenation during Pediatric Cardiac Surgery: Identification of Vulnerable Periods using Near-infrared Spectroscopy. Res Inno Anaesth 2016;1(1):15-18.

Source of support: Nil

Conflict of interest: None

\footnotetext{
${ }^{1}$ Resident (2nd Year), ${ }^{2}$ Professor

${ }^{1}$ Department of Cardiac Anaesthesia, Seth GS Medical College Mumbai, Maharashtra, India

${ }^{2}$ Department of Cardiothoracic Anaesthesia, Seth GS Medical College, Mumbai, Maharashtra, India

Corresponding Author: Mehul Mange, Resident (2nd Year) Department of Cardiac Anaesthesia, Seth GS Medical College Mumbai, Maharashtra, India, e-mail: drmehulmange@gmail.com
}

\section{INTRODUCTION}

In recent decades there has been a marked reduction in surgical mortality for many forms of congenital heart disease. Neurologic sequelae remain, however, a wellrecognized and potentially devastating complication of pediatric cardiac surgery. ${ }^{1}$ These complications appear to occur more commonly after deep hypothermic circulatory arrest. ${ }^{2}$ Although the etiology of neurologic injury is almost certainly multifactorial, imbalance between cerebral oxygen supply and demand is likely to play an important role. The ability to monitor cerebral oxygenation during, and immediately after, cardiac surgery offers the possibility for predicting and preventing neurological injury. $^{2}$

In this study we have used near-infrared spectroscopy to help identify periods of mismatch between cerebral oxygen supply and demand during pediatric cardiac surgery.

\section{MATERIALS AND METHODS}

Following institutional approval and informed parental consent, 18 children were monitored during cardiac surgery. Their diagnosis and procedures are shown in Table 1. A total of 18 procedures were carried out on cardiopulmonary bypass.

General anesthesia used a standard technique involving high-dose opioid induction: fentanyl $10 \mu \mathrm{g} /$ $\mathrm{kg}$, midazolam $0.1 \mathrm{mg} / \mathrm{kg}$, muscle relaxant rocuronium $1 \mathrm{mg} / \mathrm{kg}$. Maintenance was with $0.5-1 \%$ isoflurane in air and oxygen. Ventilation was adjusted to maintain normocarbia, as assessed by end-tidal $\mathrm{CO}_{2}$ sampling and intermittent arterial blood gas analysis. Cardiopulmonary bypass utilized a nonpulsatile roller pump and membrane oxygenator. Standard pump flow rates were 100-120 ml/ $\mathrm{kg} / \mathrm{min}$, although in selected patients periods of low-flow bypass were employed. Management of acid-base status was used during cardiopulmonary bypass. Near-infrared monitoring utilized the Invos 3100 cerebral oximeter.

This measures intracerebral hemoglobin oxygen saturation by spectroscopy of reflected near-infrared light. Since the cerebral microcirculation contains arterial, venous, and capillary components, the regional cerebral oxygen saturation $\left(\mathrm{rSO}_{2}\right)$ represents a weighted average 
Table 1: Patient characteristics and operative details

\begin{tabular}{llllll}
\hline Patient number & Age at operation (years) & Weight at operation $(\mathrm{kg})$ & Diagnosis & Procedure & CPB/arrest \\
\hline 1 & 12 days & 3.3 & TGA & Switch & CPB \\
2 & 2 years & 6.0 & VSD & Correction & CPB \\
3 & 1 month & 2.5 & TGA & Switch & CPB \\
4 & 5 months & 8.5 & TAPVD & Correction & CPB \\
5 & 3 months & 4.0 & Taussig-Bing & PA banding & CPB \\
6 & 8 years & 18 & VSD & Correction & CPB \\
7 & 0.8 & 8.5 & AVSD & Correction & CPB \\
8 & 1.0 & 5.3 & T of Fallot & Correction & CPB \\
9 & 1.1 & 8.0 & PAVSD & Correction & CPB \\
10 & 1.3 & 8.7 & TGA-Mustard & Switch & CPB \\
11 & 3.6 & 13.7 & AVSD & Correction & CPB \\
12 & 4.4 & 20.0 & VSD & Correction & CPB \\
13 & 4.4 & 17.7 & ASD & Correction & CPB \\
14 & 5.1 & 23.0 & ASD & Correction & CPB \\
15 & 6.2 & 17.0 & T of Fallot & Correction & CPB \\
16 & 9.1 & 22.5 & ASD & Correction & CPB \\
17 & 9.7 & 26.0 & PAVSD & Correction & CPB \\
18 & 12.5 & 36 & T of Fallot & Correction & CPB \\
\hline
\end{tabular}

AR: Aortic regurgitation; AS: Aortic stenosis; ASD: Atrial septal defect; AVR: Aortic valve replacement; AVSD: Atrioventricular septal defect; CPB: Cardiopulmonary bypass; DHCA: Deep hypothermic circulatory arrest $\left(\mathrm{B} 20^{\circ} \mathrm{C}\right)$; MR: Mitral regurgitation; MV: Mitral valve; MVR: Mitral valve replacement; PA: Pulmonary artery; PAVSD: Pulmonary atresia with ventricular septal defect; T: Tetralogy; TAPVD: Total anomalous pulmonary venous drainage; TGA: Transposition of the great arteries; VSD: Ventricular septal defect

assuming the venous component to be predominant (estimated as $75 \%$ by volume). ${ }^{3}$

The sensor consists of a near-infrared light transmitter and two photo detectors (optodes) positioned 3 and $4 \mathrm{~cm}$ from the infrared source. This arrangement allows spatial resolution since the optode nearest the light source receives a signal from the light that has traveled in an arc through superficial tissues, while the more distant optode receives light that has passed through superficial and deeper tissues. "Subtraction" processing of the two signals allows calculation of oxygen saturation of hemoglobin from cerebral tissue while minimizing "contamination" from superficial (extracerebral) sources. The cerebral oximeter displays the regional cerebral hemoglobin oxygen saturation. The light source and optodes are contained within a flexible adhesive pad, which was placed over the forehead lateral to the midline to avoid the superior sagittal sinus and at least $2 \mathrm{~cm}$ above the eyebrows to avoid the frontal sinus. Monitoring was commenced shortly after induction of general anesthesia and continued throughout the procedure until the child left the operating room.

The following variables were monitored approximately every $5 \mathrm{~min}$ during surgery, or more frequently when events were changing rapidly: $\mathrm{rSO}_{2}$, systemic arterial saturation, heart rate, mean blood pressure, central venous pressure, cardiopulmonary bypass flow rates and nasopharyngeal and esophageal temperatures. The $\mathrm{rSO}_{2}$ profiles were described in four distinct periods: prebypass, bypass, rewarming/reperfusion, and postbypass.

\section{RESULTS}

\section{Prebypass}

A total of 18 patients were analyzed (see Table 1). In five patients, $\mathrm{rSO}_{2}$ reached $35 \%(20,27,28,28$, and $33 \%)$. In three cases this fall was associated with handling and dissection of the heart (especially the superior vena cava) prior to going onto cardiopulmonary bypass. Graph 1 shows $10 \%$ mean decrease in prebypass $\mathrm{rSO}_{2}$ compared with baseline.

\section{Bypass}

The first period of bypass raised the $\mathrm{rSO}_{2}$ by a mean $11 \%$ points (Graph 3 ). During cardiopulmonary bypass, patients were cooled to between 26 and $28^{\circ} \mathrm{C}$, with three patients being cooled to less than $20^{\circ} \mathrm{C}$. The $\mathrm{rSO}_{2}$ at the time that this temperature was reached varied inversely

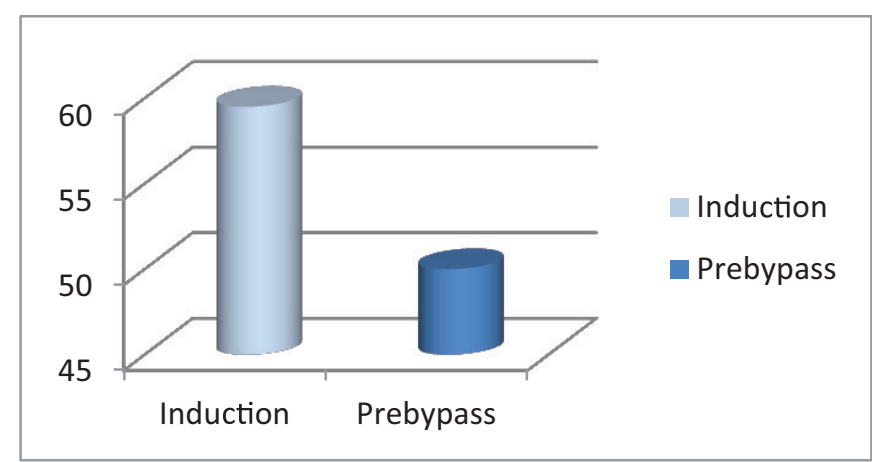

Graph 1: A 10\% mean decrease in prebypass $\mathrm{rSO}_{2}$ compared with baseline 


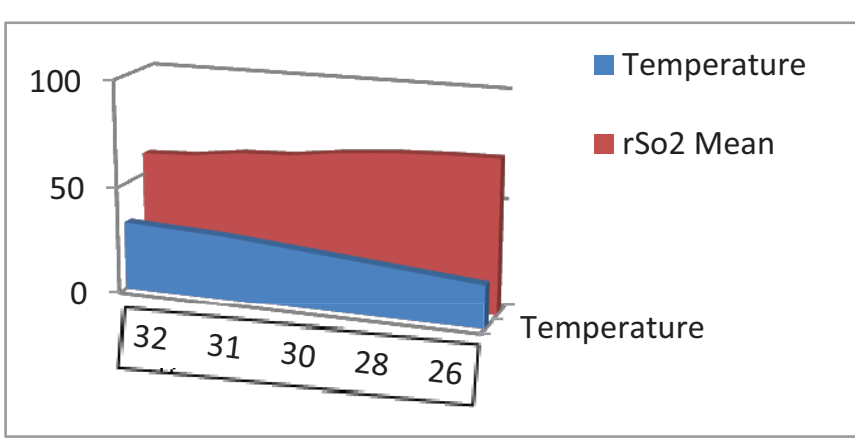

Graph 2: Inverse relation of temperature and $\mathrm{rSO}_{2}$

with rate of cooling. Graph 2 shows inverse relation of temperature and $\mathrm{rSO}_{2}$.

\section{Rewarming/reperfusion}

Rewarming on cardiopulmonary bypass for patients not undergoing circulatory arrest resulted in a universal decrease in $\mathrm{rSO}_{2}$. There was an inverse relationship during rewarming between $\mathrm{rSO}_{2}$ and nasopharyngeal temperature (Graph 3).

\section{POSTBYPASS}

Discontinuation of cardiopulmonary bypass was associated with a precipitous fall in $\mathrm{rSO}_{2}$ (Graph 4). In these cases, the $\mathrm{rSO}_{2}$ fell to less than $40 \%$. This fall appeared to be due to poor cardiac output with low systemic

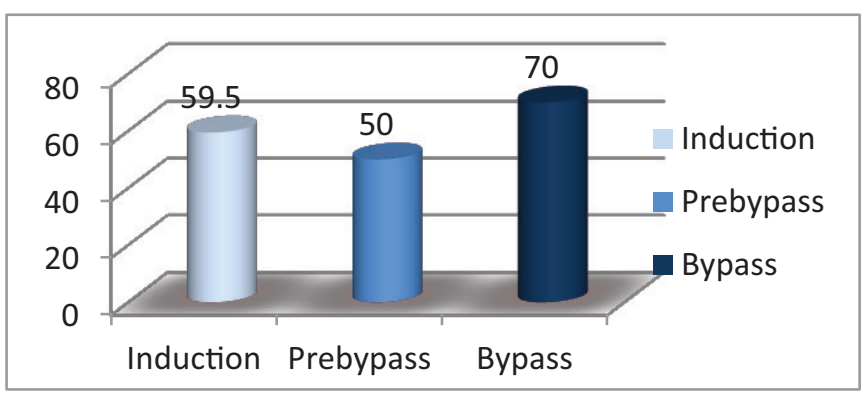

Graph 3: Mean values of $\mathrm{rSO}_{2}$ during induction prebypass and bypass

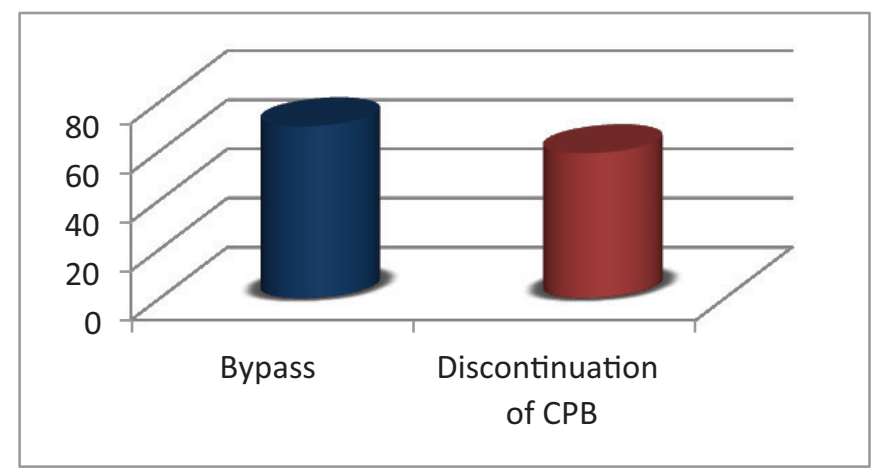

Graph 4: Fall of mean $\mathrm{rSO}_{2}$ while discontinuation of cardiopulmonary bypass arterial pressure in the presence of normothermia or during rapid rewarming.

\section{DISCUSSION}

Although neurologic injury during pediatric cardiac surgery is almost certainly multifactorial in origin, it is self-evident that cerebral oxygen supply must meet metabolic demands. It is known that cooling reduces cerebral metabolism and that the cerebral metabolic rate falls exponentially with temperature reduction. ${ }^{4}$ By contrast, cerebral blood flow falls linearly with cooling. Thus, induced hypothermia should result in progressive rise in the ratio of cerebral blood flow to metabolism. It might, therefore, be expected that cardiopulmonary bypass under hypothermic conditions should not, in itself, result in inadequate oxygen delivery.

Greeley et $\mathrm{al}^{4}$ have recently emphasized the need for study of all periods during pediatric cardiac surgery and not just the period of circulatory arrest. The "preparation period" prior to onset of circulatory arrest may be a vital determinant of how well circulatory arrest is tolerated. ${ }^{4}$ Important factors at this time might include method and rate of cooling, as well as strategy for acidbase regulation. The early postbypass and postoperative periods might also be times of increased vulnerability for hypoxic-ischemic damage.

Following profound hypothermia, cerebral autoregulation may remain absent or impaired for several hours. If cardiac output is inadequate following discontinuation of cardiopulmonary bypass, then cerebral oxygen delivery may become insufficient to meet metabolic demands during and after rewarming. It is clear that any tool which can assess the cerebral oxygen supply/demand relationship may have major potential for identifying the most vulnerable periods for ischemic damage during and after pediatric cardiac surgery. Tools for the assessment of cerebral blood flow (e.g., xenon clearance) and metabolism during cardiopulmonary bypass have been well described, but are cumbersome and not well suited for continuous monitoring in a clinical setting.

Cerebral desaturation was observed in several patients during the prebypass period. This was most commonly associated with dissection around the superior vena cava and caval cannulation. Whether this insult is sufficient to cause cerebral damage remains unclear, but it offers the option of adjusting surgical technique if profound falls in $\mathrm{rSO}_{2}$ occur. Furthermore, we identified one case in which $\mathrm{rSO}_{2}$ monitoring (in retrospect) offered early clues to impending cardiovascular collapse prior to change in hemodynamic parameters. In almost all patients, institution of cardiopulmonary bypass with simultaneous cooling resulted in a rise in $\mathrm{rSO}_{2}$, as might be expected 
based on our understanding of the cerebral response to cooling. These observations are also consistent with changes in jugular bulb saturation during hypothermic cardiopulmonary bypass. Since standard temperature monitoring during cardiopulmonary bypass does not reliably predict efficiency of cerebral cooling, near-infrared spectroscopy may prove to be a useful technique for assessing adequacy of cerebral protection in individuals as well as a suitable research tool for comparing differing strategies of cerebral protection (e.g., differing cooling techniques, optimal management of acid-base status).

Cerebral oxygenation cannot be assessed during circulatory arrest by jugular bulb monitoring. Although magnetic resonance spectroscopy can provide information about high-energy phosphorus compounds in the brain, 3,5 bedside monitoring is unavailable and therefore this technique is not suitable for clinical use during cardiac surgery. Near-infrared spectroscopy is thus a very attractive tool for continuous monitoring during deep hypothermic circulatory arrest as the technique is noninvasive, safe, and simple to perform in the operating room.

At the present time, it is impossible to predict the "safe period" for circulatory arrest in any individual. It seems possible that assessment of cerebral oxygenation at the onset of deep hypothermic circulatory arrest by near-infrared spectroscopy, combined with continuous monitoring during deep hypothermic circulatory arrest, might help predict when a patient is at risk for the development of hypoxic-ischemic cerebral injury.

Our observations support the contention of other authors that the period early after discontinuation of cardiopulmonary bypass may be a vulnerable ${ }^{4}$ period for provision of adequate oxygen delivery to meet cerebral metabolic demands. During this period cerebral metabolic rate will rise, but ability to increase oxygen delivery may be impaired. This may be particularly problematic following deep hypothermic circulatory arrest when cerebral autoregulation may remain impaired for a variable period of time after discontinuation of cardiopulmonary bypass. In the presence of poor ventricular function and systemic hypotension, cerebral oxygen delivery may be compromised. The ability to assess noninvasively the cerebral oxygen supply/demand relationship at this critical time might allow for modifications in clinical management. For example, one might adjust the rate of weaning from cardiopulmonary bypass, rewarm more judiciously, or augment inotropic support to prevent precipitous fall in $\mathrm{rSO}_{2}$.

\section{CONCLUSION}

These preliminary observations suggest that pre- and early postbypass are vulnerable periods for maintenance of cerebral oxygenation during pediatric cardiac surgery and that near-infrared spectroscopy is a promising tool for monitoring the cerebral oxygen supply/demand relationship, especially during circulatory arrest when other monitoring modalities are unavailable.

Use of near-infrared spectroscopy remains a research tool in the setting of congenital heart surgery. An appreciation of potential vulnerable periods for neurologic injury has led us, however, to examine more critically current practices. Various strategies may be employed to reduce the risk of cerebral injury. These include a longer period of cooling on cardiopulmonary bypass prior to instituting circulatory arrest, increased usage of superficial cooling of the head with ice packs, avoidance of "warm" $\left(20^{\circ} \mathrm{C}\right)$ circulatory arrest, and short periods of intermittent reperfusion during long episodes of deep hypothermic circulatory arrest. Near-infrared spectroscopy offers a suitable technique to assess whether these strategies can help maintain adequate cerebral oxygenation.

\section{LIMITATIONS}

Several technical considerations remain to be explored in greater detail. These include the possibility of "contamination" of signal by superficial extracerebral tissues (perhaps less problematic in the pediatric age group), the validity of assumptions about the constancy of the path length of light in the rapidly changing conditions (e.g., alterations in hematocrit) encountered during cardiopulmonary bypass and circulatory arrest, and the possibility that intravascular oxygenation may be an inadequate surrogate for intracellular oxygenation. Ultimately, the potential of this technique for predicting neurologic injury during pediatric cardiac surgery can only be established by large-scale, long-term prospective clinical trials.

\section{ACKNOWLEDGMENT}

We thank our Dean Dr. Avinash Supe, Seth GS Medical College, Mumbai, Maharashtra, India.

\section{REFERENCES}

1. Ferry PC. Neurologic sequelae of cardiac surgery in children. Am J Dis Child 1987 Mar;141(3):309-312.

2. Wernovsky G, Jonas RA, Hickey PR, du Plessis AJ, Newburger JW. Clinical neurologic and developmental studies after cardiac surgery utilizing hypothermic circulatory arrest and cardiopulmonary bypass. Cardiol Young 1993 Jul;3(3):308-316.

3. Delpy DT, Cope MC, Cady EB, Wyatt JS, Hamilton PA, Hope PL, Wray S, Reynolds EOR. Cerebral monitoring in newborn infants by magnetic resonance and near infrared spectroscopy. Scand J Clin Lab Invest 1987;47(Suppl 188):9-17.

4. Greeley WJ, Kern FH, Mault JR, Skaryak LA, Ungerleider RM. Mechanisms of injury and methods of protection of the brain during cardiac surgery in neonates and infants. Cardiol Young 1993 Jul;3(3):317-30.

5. Jonas RA. Experimental studies of hypothermic circulatory arrest and low flow bypass. Cardiol Young 1993 Jul;3(3):299-307. 